\title{
Performance of Energy Detector for Cognitive Radio System over AWGN and Rayleigh Channel
}

\author{
Buthaina Mosa, PhD \\ Electric and communication Engr. Dept. \\ College of Engineering \\ University of Baghdad
}

\author{
Aya Falah Algamluoli \\ Electric and communication Engr. Dept. \\ College of Engineering \\ University of Baghdad
}

\begin{abstract}
Cognitive radio has a critical application which it is Spectrum access, and knowing that the key to this application is detected in the spectrum to find free bands. Nowadays the studies confirmed that the energy detector methods are the most convenient method. In this work, we present the energy detector and explain how it is a convenient method in a sense because it doesn't need any prior information about the primary user. The simulation of energy detection methods has been done in MATLAB program, for both, AWGN and Rayleigh channels. The simulation confirmed the theoretical results, which gives that the performance of AWGN channel is greater than a Rayleigh channel, it is also verified that the performance of the detector is independent of the type of modulation.
\end{abstract}

\section{Keywords}

Cognitive radio, spectrum sensing, energy detection, AWGN channel, Rayleigh channel, probability of detection, probability of false alarm.

\section{INTRODUCTION}

Nowadays, using of the frequency spectrum is becoming increasingly demanded. The present management of the frequency spectrum cannot boost the rise requirement of wireless services and different technologies. These problems are limited by Cognitive radio (CR). CR is introduced by Mitola and, it increased spectral efficiency [1]. Recently, it is a newly emerging technology which has been proposed to implement some kind of intelligence. It can automatically sense, recognize, and make wise use of any available radio frequency spectrum. It is proposed for sharing the license spectrum to unlicensed users without harmful interference to licensed system [2]. In CR terminology, the higher priority rights on the usage of a licensed spectrum belong to the primary user (PU). And lower priority to secondary user (SU). SU can utilize the free band of spectrum in such a way to do not cause interference to PUs. So, SUs need to have cognitive radio facility, such as sensing the spectrum in reliably to check whether it is being used by a PU or not and to change the radio parameters to utilize the free band of the spectrum [3].

Spectrum sensing is an exceptionally important task in a CR system. The transmissions of PUs have to be reliably detected. Thus, spectrum sensing is the first stage towards adaptive transmission in free spectral bands. Without harm PU or cause any interference to PU, the secondary system has to be aware of the spectrum to exploit the available spectrum efficiently. There are certainly a number of approaches that can be used to check whether the PU's signal is present or not, but the only autonomous and flexible approach is based on measurements of the actual occupancy in given location and time [4]. Many different methods are offered for spectrum sensing. These methods are proposed for recognizing the presence of signal transmission. These techniques are mainly categorized into two families: Blind sensing techniques and signal specific sensing techniques while the first don't need any prior knowledge about the transmitted signal which it is the PU. While the second type needs some information about the characteristics of the PU such as carrier frequency, modulation type, symbol period, and etc. This classification leads to decide whether one of these choices best fit the CR [5]. The most known methods for spectrum sensing to detect the band are Energy detection method, Cyclostationary feature detection, and Matched filter detection.

Because of a simple implementation of energy detector and it has low computational complexity.it is widely used. Also, this method needs to estimate only the noise power to set the threshold value and does not require any information about the PU. This makes energy detection based sensing a semiblind technique [6]. Being the focus of this paper, on the methods of spectrum sensing "Energy detector", analysis, and study parameter effect on performance. Finally, study the effect of PU in the performance of the detector.

The metrics of detection performance are the probability of detection and false alarms. The probability that a SU declares that a PU is present when the spectrum is idle is called the probability of a false alarm (PFA). Conversely, the probability that the SU declares that the PU is present when the spectrum is occupied by the PU is called the probability of detection (PD). The probability of misdetection (PMD) indicates the probability that the SU declares that the PU is absent when the spectrum is occupied. CR should exhibit a low probability of false alarm and a high probability of detection. Misdetection leads to interference with the PUs, while false alarms decrease the efficiency of spectrum utilization. Complexity of the mechanism is another issue. Since CRs are envisioned to operate on wireless nodes with small size and power, the spectrum sensing [7].

The details of this paper as follows, section 2 deals with the spectrum sensing expression. Whereas section 3 deals with the analyses of the energy detection in. In Section 4 deals with the simulation in MATLAB program. Section 5 the result of simulation and present paragraph of different parameter that effect on performance of energy detector. Finally, section 6 concludes the paper.

\section{SPECTRUM SENSING EXPRESSION}

A critical component of cognitive radio system is this "spectrum sensing". The SU should sense the spectrum efficiently, quickly seize opportunities to transmit, and vacate the spectrum should a PU reoccupy the spectrum.

As noted in, a critical component of opportunistic spectrum allocation is the design of the spectrum sensor for opportunity detection. The practical system which is commonly used in 
CR is transmitter detection. Transmitter detection approach is based on the detection of the weak signal from a PU. To achieve dynamic spectrum sharing, the CR transmitter should have capability to determine if a signal from PU is locally present in a certain spectrum. Basic hypothesis model for transmitter detection can be defined as follows

$y(t)= \begin{cases}n(t) \quad ; & \mathcal{H} 0 \\ h s(t)+n(t) ; & \mathcal{H} 1\end{cases}$

Where $y(t)$ is the signal received to the cognitive user, $\mathrm{s}(\mathrm{t})$ is the transmitted signal of the PU, $\mathrm{n}(\mathrm{t})$ is the additive white Gaussian noise and $\mathrm{h}$ is the amplitude gain of the channel. $\mathcal{H} 0$ Is a null hypothesis, which states that there is no licensed user signal in a certain spectrum band. On the other hand, $\mathcal{H} 1$ is an alternative hypothesis, which indicates that there exists some licensed user signals [8]. The performance of a detector is characterized by using the pair (PD, PFA),

$P D=P($ decide of $\mathcal{H} 1 \mid \mathcal{H} 1)$

$P F A=P($ decide of $\mathcal{H} 1 \mid \mathcal{H} 0)$

The calculation of PD and PFA will allow us to plot the curve $\mathrm{PD}=f(\mathrm{PFA})$ which is called Receiver Operating Characteristic (ROC) and it is the performance of detector [1].

\section{ENERGY DETECTOR}

Energy detection is a naive signal detection approach which is referred in classical literature as radiometry. Energy detector is especially suitable for wideband spectrum sensing when CR doesn't have sufficient information about the PU signal [6].

Figure 1 depicts the conventional principle of energy detector; at first the input signal is filtered with a Band Pass Filter (BPF) to select the bandwidth. Next the filtered signal is squared and integrated over the observation interval. Then the output of the integrator is compared to a threshold to decide whether if the primary user is present or not [9].

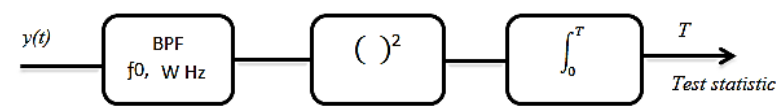

Fig 1: The conventional energy detector

The test statistic for the conventional energy detector is:

$T=\frac{1}{N 0} \int_{0}^{T} y^{2}(t) d t=\sum_{i=1}^{2 T W} y_{i}^{2}$

The output of block, $T$, follows chi-square distribution,

$T \sim \begin{cases}X_{2 T W}^{2} ; & H 0 \\ X_{2 T W}^{2}(2 \gamma) ; & H 1\end{cases}$

Where N0 is the one-sided noise power spectral density, $X_{2 T W}^{2}$ and $X_{2 T W}^{2}(2 \gamma)$ represent central and conditionally noncentral chi-square distribution respectively, with $2 T W$ degrees of freedom and $2 \gamma$ is a non-centrality parameter for the distribution, $\gamma$ is an instantaneous SNR and ,TW, is timebandwidth product is an integer number [1]. "The most often used approaches in the energy detection are based on the Neyman-Pearson (NP) lemma". The NP detection criterion enlarges the PD for a given PFA.

Under Additive white Gaussian noise (AWGN) channel the PFA can be computed as,

$\mathrm{PFA}=\mathrm{P}(\mathrm{T}<\lambda)=\frac{\Gamma\left(\mathrm{U}, \frac{\lambda}{2}\right)}{\Gamma(\mathrm{U})}$
Where $\Gamma($.$) and \Gamma(.,$.$) are complete and incomplete gamma$ function respectively.

The PD calculate as follow,

$P D=P(T>\lambda)=Q_{u}(\sqrt{2 \gamma}, \sqrt{\lambda})$

Where $\gamma=\frac{\sigma_{s}^{2}}{\sigma_{w}^{2}}, Q_{u}(.,$.$) is the generalized Marcum Q$-function with order U[10].

In [11], According to the central limit theorem if the number of test is rather large (U> 250), the law of $\chi^{2}$ can be approximated by a normal distribution, Figure 2 explain the conventional energy detector when use digital communication.

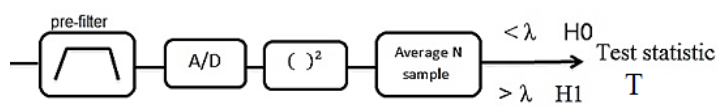

Fig 2: Conventional energy detector with digital

After convert receive signal to digital we assume it have $\mathrm{N}$ samples data denoted by $y_{i}$ where $i=1 \ldots . . N$ and the test statistic will be

$T=\sum_{N}(y(n))^{2}$

And the distribution will convert to normal distribution will follow as

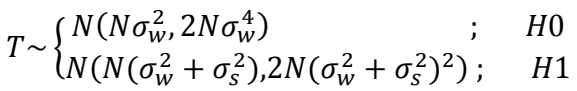

The metrics of performance will change to,

$$
\begin{aligned}
P F A & =Q\left[\frac{\lambda-N \sigma_{w}^{2}}{\sqrt{2 N \sigma_{w}^{4}}}\right] \\
P D & =\left[\frac{\lambda-N\left(\sigma_{w}^{2}+\sigma_{s}^{2}\right)}{\sqrt{2 N\left(\sigma_{w}^{2}+\sigma_{s}^{2}\right)^{2}}}\right]
\end{aligned}
$$

In [12], over fading statistics, as Rayleigh fading channel the signal amplitude follows a Rayleigh distribution, the $\gamma$ follows an exponential law which is the probability density function(pdf) given by:

$f_{y}(\gamma)=\frac{1}{\gamma} \exp \left(-\frac{\gamma}{\gamma}\right)$

Where $\bar{\gamma}$ is mean value of $\gamma$, or $\bar{\gamma}_{\gamma}=\mathrm{E}(\gamma)$ with $\mathrm{E}($.$) denotes$ the mathematical expectation.

In order to find the probability of detection over Rayleigh channel $\left(P D_{\text {Ray }}\right)$, by averaging equation (13) over equation (18) as follow,

$P D_{\text {Ray }}=\int Q_{u}(\sqrt{2 \gamma}, \sqrt{\lambda}) f_{y}(\gamma) d \gamma$

By substitute (18) the equation will become,

$P D_{\text {Ray }}=\exp ^{-\frac{\lambda}{2}} \sum_{K=0}^{\frac{U}{2}-1} \frac{1}{K !}\left(\frac{\lambda}{2}\right)^{K}+\left(\frac{1+\bar{\gamma}}{\bar{\gamma}}\right)^{\frac{U}{2}-1} *\left[\exp ^{-\frac{\lambda}{2(1+\bar{\gamma})}}-\right.$

$\left.\exp ^{-\frac{\lambda}{2}} \sum_{K=0}^{\frac{U}{2}-1} \frac{1}{K !}\left(\frac{\lambda_{\bar{\gamma}}}{2\left(1+\frac{-}{\gamma}\right)}\right)^{K}\right]$

\section{MATLAB SIMULATION}

The simulation of PD is investigated in order to find the performance of the spectrum sensing methods detector. It will be made by using model which consists three parts. In the first part, the generation of a signal which we can chose the PU signal. In the second, a transmission channel and we chose 
two types AWGN and Rayleigh. In the last part, the receiver will contain our sensing methods and it is called CR.

Figure 3 illustrates the algorithm that used in MATLAB to simulate performance of energy detector. We used monteCarlo simulation to find PD.

Where N: Presents the number of samples, itre: Presents the number of iteration to calculate the $\mathrm{Pd}$ and

Nod: presents the number of detection signal. The simulation will did for a fixed value of SNR.

\section{RESULT AND DISCUSSION}

The simulation has been done according to algorithms with different set of parameters to show effect of these parameters on performance. Begin with AWGN channel and setting value of number of samples, SNR and degree of freedom of detector.

Figure 4 shows performance of energy detector with $\mathrm{N}=840$, 1400, 2100, 7700, 14700, 21700, 28700, 42700, 140700 and $\mathrm{SNR}=-32 \mathrm{~dB}$ as expected, performance improves monotonically with increasing $N$.

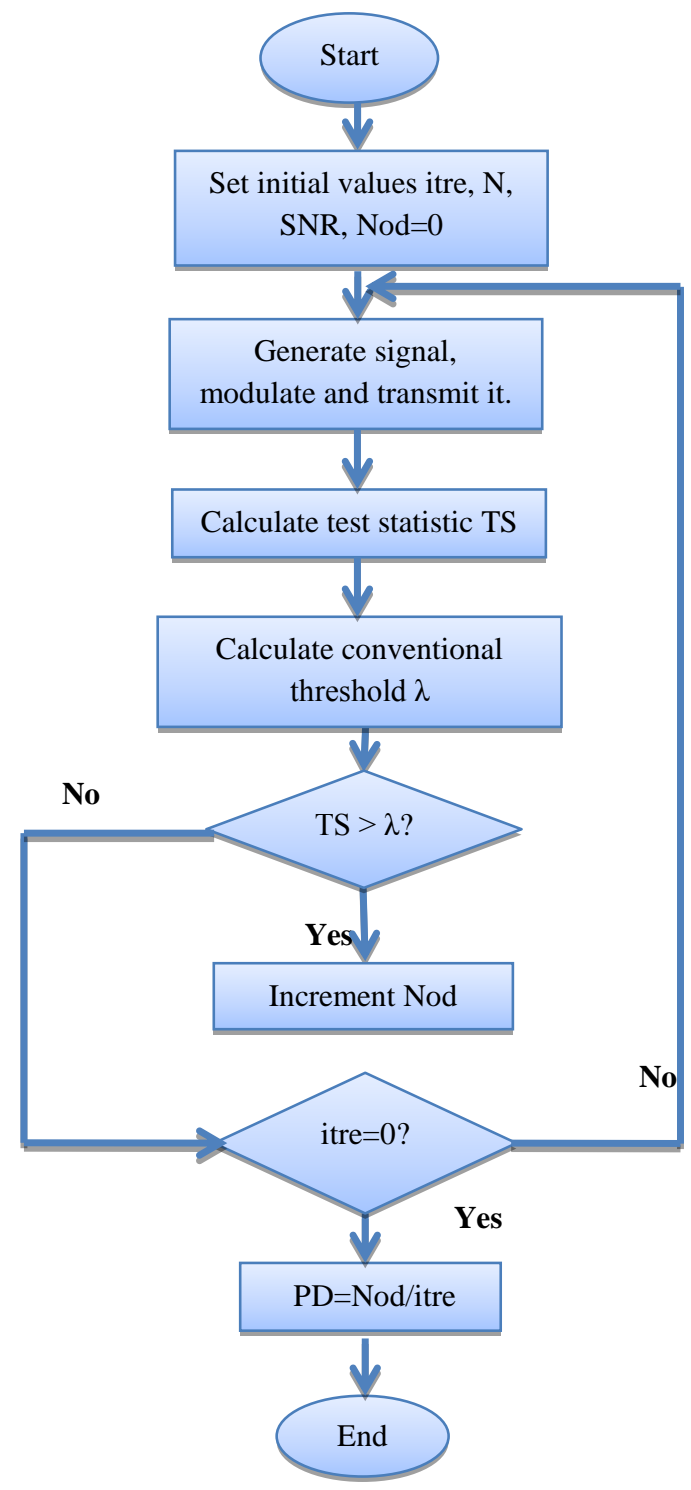

Fig 3: simulation of PD to get performance

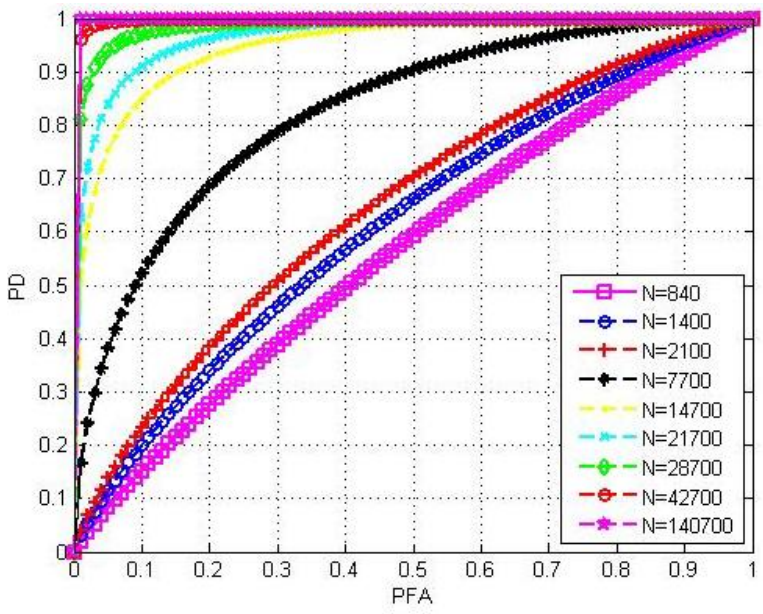

Fig 4: ROC for energy Detector using different values of number of sample and $\mathrm{SNR}=-30 \mathrm{~dB}$

Figure 5 shows performance of energy detector with $\mathrm{SNR}=$ $34,-32,-30,-28,-26,-24,-22,-20$ and $\mathrm{N}=2480$ as expected, performance improves monotonically when SNR increase

Figure 6 shows effect of SNR and $\mathrm{N}$ both parameters on performance of energy detector. However, we must take into account the side effects had to heating the card reception, which will create an internal noise. And, the second factor is SNR, which is an external element that will depend on the received conditions.

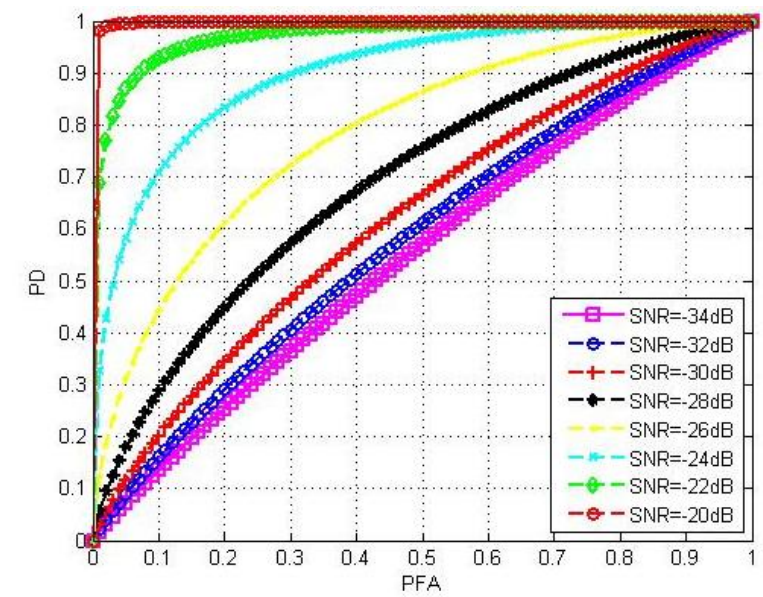

Fig 5: ROC for energy Detector using different values of SNR and number of sample $=\mathbf{2 4 8 0}$

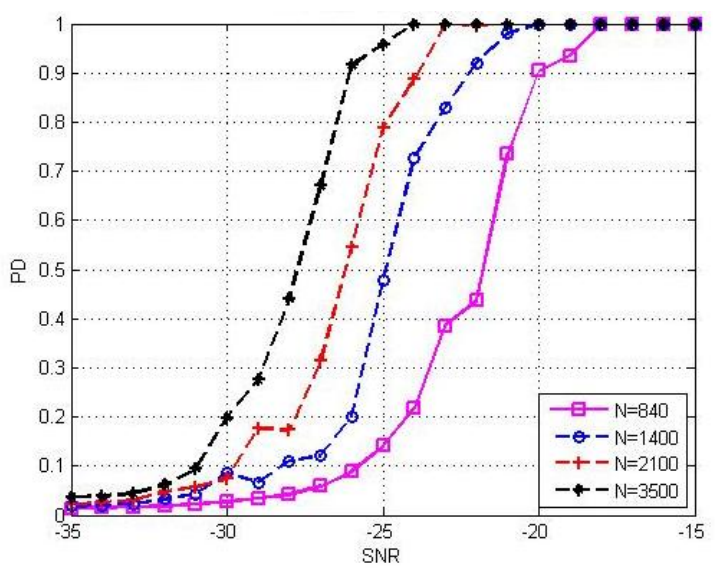

Fig 6: plot SNR VS PD for energy Detector with PFA=0.1 
Figure 7 show performance of energy detector with $U=1,2,3$ and $\mathrm{SNR}=1 \mathrm{~dB}$ as expected, performance increase with $\mathrm{U}$ increase. And Figure 8 show performance of energy detector with $\mathrm{U}=2$ and $\mathrm{SNR}=-15,-10,1 \mathrm{~dB}$ as expected, performance increase with SNR increase. However from these figures, it is clear that for a low value of the PFA, the PD is closer to zero. When PFA begins to increase as well as PD increases.

Figure 9 shows that the performance of the energy detector under both AWGN and Rayleigh channels with SNR=-10dB. According to Figure 10 can see that the type of modulation does not have a great influence on the quality of detection.

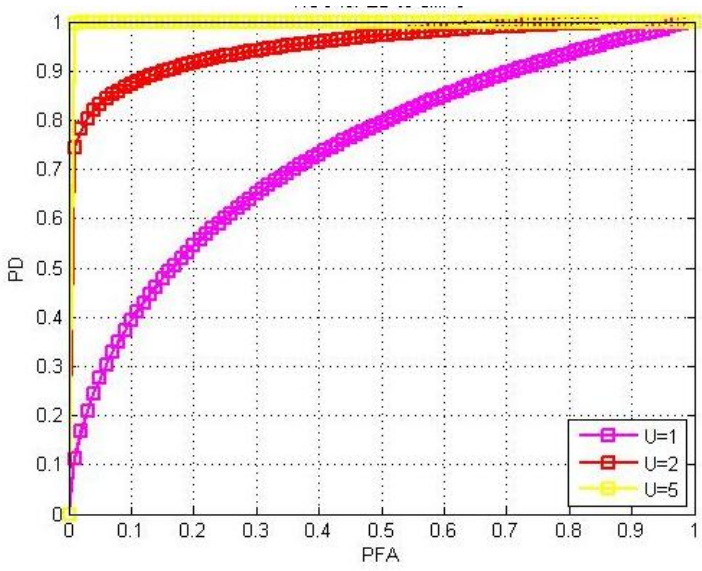

Fig 7: ROC for energy Detector using different values of degree of freedom and $\mathrm{SNR}=1 \mathrm{~dB}$

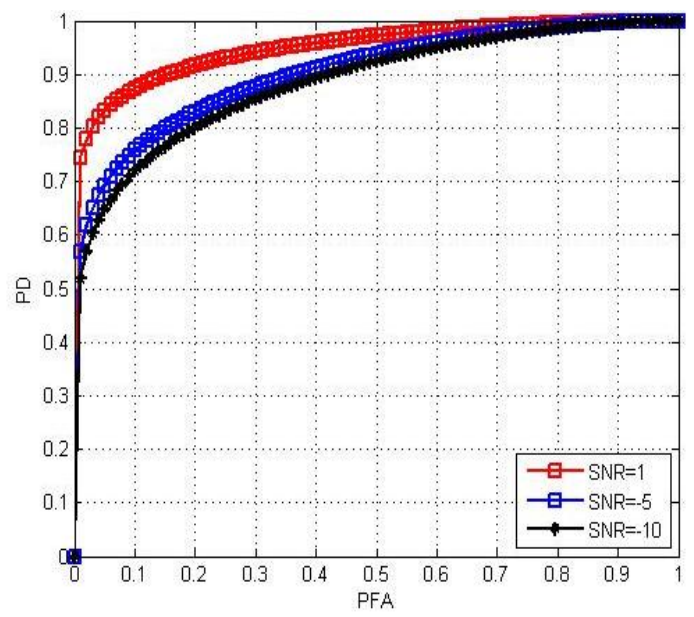

Fig 8: ROC for energy Detector using different values of SNR and $U=2$

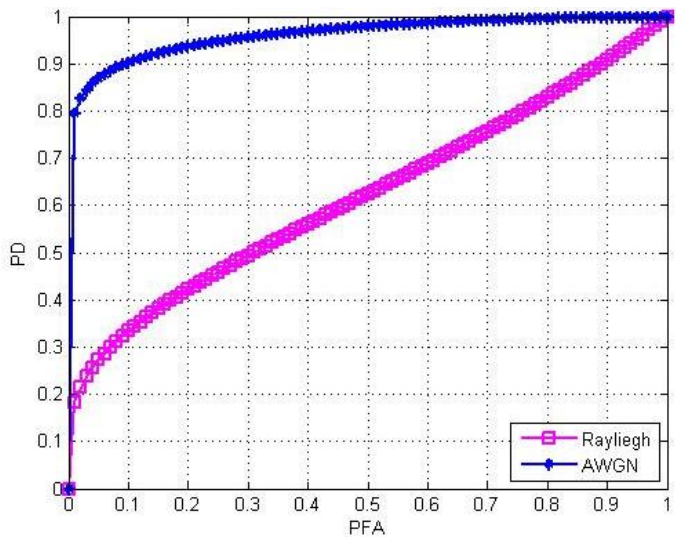

Fig 9: ROC for energy Detector under AWGN and Rayleigh channels

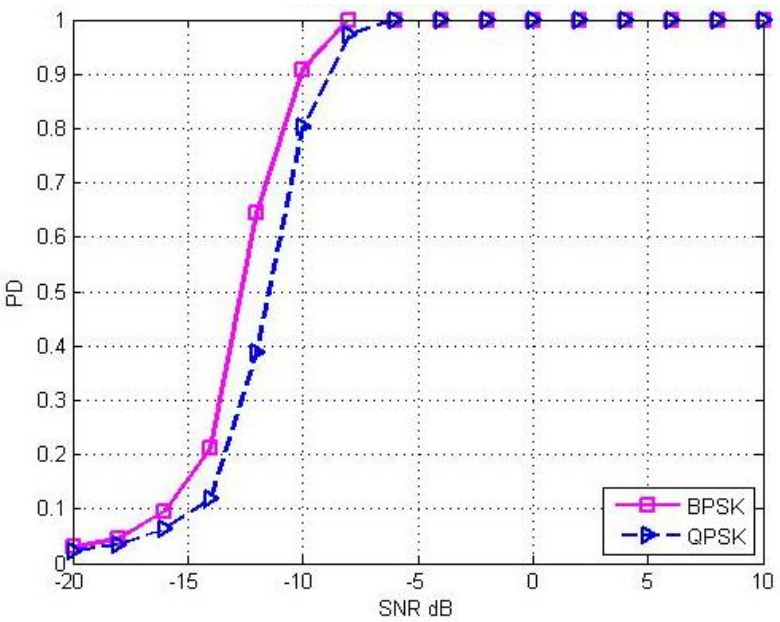

Fig 10: ROC for energy Detector with different modulation using BPSK and QPSK under AWGN channel

\section{CONCLUSION}

Cognitive radio has been proposed as an optimal solution to improve the frequency underutilization. The stage of spectrum sensing is the first and the most important function of the cognitive radio. One of the most used techniques to detect the holes in the frequency spectrum is the energy detection. In this respect, we have analyses analytically the performance of the energy detection using the Receiver Operating Characteristics curve in both AWGN and Rayleigh channels; Simulation results show that the performance depend on number of sample , SNR, degree of freedom and when it increase the performance increase too. But it is independent on the type of modulation.

\section{REFERENCES}

[1] Abdessamad, E., Saadane, R., El Aroussi, M., Wahbi, M., \& Hamdoun, A. (2014, April). Spectrum sensing with an improved Energy detection. In Multimedia Computing and Systems (ICMCS), 2014 International Conference on (pp. 895-900). IEEE.

[2] Wang, W. (2009). Cognitive radio systems. 1st ed. SL: Sciyo.com.

[3] Yucek, T., \& Arslan, H. (2009). A survey of spectrum sensing algorithms for cognitive radio applications. IEEE communications surveys \& tutorials, 11(1), 116-130.

[4] Kamil, N. H., \& Yuan, X. (2010). Detection proposal schemes for spectrum sensing in cognitive radio. Wireless Sensor Network, 2(05), 365.

[5] Zayen, B., Hayar, A., Debbabi, H., \& Besbes, H. (2009, October). Application of smoothed estimators in spectrum sensing technique based on model selection. In Ultra Modern Telecommunications \& Workshops, 2009. ICUMT'09. International Conference on (pp. 1-4). IEEE.

[6] Umar, R., \& Sheikh, A. U. (2013). A comparative study of spectrum awareness techniques for cognitive radio oriented wireless networks. Physical Communication, 9, 148-170. 
[7] Ariananda, D. D., Lakshmanan, M. K., \& Nikookar, H. (2009, May). A survey on spectrum sensing techniques for cognitive radio. In Cognitive Radio and Advanced Spectrum Management, 2009. CogART 2009. Second International Workshop on (pp. 74-79). IEEE.

[8] Wyglinski, A., Nekovee, M. and Hou, Y. (2010). Cognitive radio communications and networks. 1st ed. Amsterdam: Elsevier.

[9] Ghasemi, A., \& Sousa, E. S. (2005, November). Collaborative spectrum sensing for opportunistic access in fading environments. In New Frontiers in Dynamic Spectrum Access Networks, 2005. DySPAN 2005. 2005 First IEEE International Symposium on (pp. 131-136). IEEE.
[10] Yue, W. J., Zheng, B. Y., Meng, Q. M., \& Yue, W. J. (2010). Combined energy detection and one-order cyclostationary feature detection techniques in cognitive radio systems. The Journal of China Universities of Posts and Telecommunications, 17(4), 18-25.

[11] Cabric, D., Tkachenko, A., \& Brodersen, R. W. (2006, August). Experimental study of spectrum sensing based on energy detection and network cooperation. In Proceedings of the first international workshop on Technology and policy for accessing spectrum (p. 12). ACM.

[12] Ranjeeth, M., \& Anuradha, S. (2015). Performance of Fading Channels on Energy Detection Based Spectrum Sensing. Procedia Materials Science, 10, 361-370. 